\title{
OC16 - Calculation skills and e-learning platform: study pre-post test on students of paediatric nursing in Italy
}

\author{
Marilena Bambaci (Italy) ${ }^{1}$; Federica Vignali (Italy) ${ }^{1}$; Fulvio Ricceri \\ $\left(\right.$ Italy) ${ }^{2}$; Anna Persico (Italy)1; Maria Chiara Ariotti (Italy)'; \\ Liliana Vagliano (Italy) ${ }^{1}$
}

${ }^{1}$ University of Turin; ${ }^{2}$ Unit of Epidemiology, Regional Health Service ASL TO3

Theme: Child protection and managing risk.

Keywords: Nursing student, skill calculation, mathematical error, drug calculation, children.

Errors in medication is a very recurring problem in paediatric units and for that reason paediatric nursing students have to possess good mathematical skills (Doherty C, 2012). It is important to evaluate these competences during their university nursing degree (Harvey 2009). This problem has been discussed and it was subsequently decided to introduce 'Clinical and Practical pharmacology' lessons like a mandatory subject.

Aim: Evaluate the paediatric nursing students learning curve before and after 'Clinical and Practical pharmacology' lessons, job placement and through an e-learning platform.

Methods: A before and after experimental study, using a non-randomized test, administered at different academic phases was used.

Results: Learning curve had an increase from $54.09 \%$ to $82.65 \%$. In particular, that result is significant before 'Clinical and Practical pharmacology' lessons and at final test $(\mathrm{p}<0.00578)$.

Conclusion: These study results are helpful for increased training action and to develop a customized e-learning platform.

\section{OC17 - Merging the person and the illness: the lived experience of emerging adults with childhood onset chronic illness}

Siobhan Macdermott (Ireland) ${ }^{1}$; Pamela Hussey (Ireland) ${ }^{1}$

${ }^{1}$ Dublin City University

Theme: Transition of care.

Keywords: Childhood onset chronic illness, illness narratives, phenomenology.

Chronic illness is emerging as major health problem in the developed world. This study explored the lived experiences of emerging adults who have lived with chronic illness since childhood in Ireland. Emerging adults often fare worse than adolescents in many areas, including adherence to treatment, and are more likely to have social, emotional and behavioural problems than their healthy counterparts. A phenomenological approach 
provided the methodology for studying of the lived experience of 15 participants aged 18 and 25 years living in Ireland who had a self-reported chronic illness since childhood. Drawing on the philosophy of van Manen's lifeworld existentials, the lived experiences were revealed. Emerging adults struggle to achieve a sense of identity which is often sabotaged by illness. The findings of this study conclude that a sense of connectedness with peers was important for emerging adults with illness as they journeyed towards developing a sense of identity.

\section{OC18 - Neurodevelopmental outcomes following congenital heart surgery}

Eleni Syrgani (Greece) $)^{1,2}$; Konstantinos Petsios (Greece) $)^{1,2}$

${ }^{1}$ National Kapodistrian University of Athens; ${ }^{2}$ 'Onassis' Cardiac Center-PICU

Theme: Complex health care and chronic disease management.

Keywords: Congenital heart disease, developmental disabilities, outcome, neurodevelopment delay.

Introduction: Congenital heart disease (CHD) may have a very important impact on central nervous system function and neurodevelopment.

Purpose: Critical evaluation of literature concerning the neurodevelopmental outcomes following congenital heart surgery.

Methods: Systematic review of 28 articles published after 2000 in PubMed, Scopus and CINAHL.

Results: Neurodevelopment morbidities can have a negative impact on early childhood development, academic performance and later transition to adulthood. The spectrum of neurodevelopment impairment is wide, depending on the complexity of $\mathrm{CHD}$ and it is characterized by mild cognitive impairment, impaired social interaction, and impairments in core communication skills, including pragmatic language, as well as inattention, impulsive behavior and impaired executive function. A number of important determinants are presented.

Conclusions: Children with CHD are at increased risk for neurodevelopmental delay. Screening and evaluation of neurodevelopmental delay, along with regular follow up, are essential steps to guide appropriate interventions to optimize their overall development.

\section{OC19 - Measuring feasibility, reliability and validity of the Greek version of PedsQL cardiac module}

Maria Drakouli (Greece) ${ }^{1,2}$; Konstantinos Petsios (Greece) ${ }^{1,2}$;

Vasiliki Matziou (Greece) ${ }^{1}$

${ }^{1}$ National and Kapodistrian University of Athens; ${ }^{2}$ Onassis Cardiac Center-PICU 